\title{
Du théâtre à la bande dessinée
}

Adapter une farce médiévale en BD au collège : quels enjeux et quelle démarche?

From theater to comic strip. Adaptation of a medieval farce for the comic book in middle school: issues and process

\section{Armelle Hesse-Weber}

\section{(2) OpenEdition}

\section{Journals}

Édition électronique

URL : http://journals.openedition.org/pratiques/3617

DOI : 10.4000/pratiques.3617

ISSN : 2425-2042

Éditeur

Centre de recherche sur les médiations (CREM)

\section{Référence électronique}

Armelle Hesse-Weber, « Du théâtre à la bande dessinée », Pratiques [En ligne], 175-176 | 2017, mis en ligne le 22 décembre 2017, consulté le 19 avril 2019. URL : http://journals.openedition.org/ pratiques/3617 ; DOI : 10.4000/pratiques.3617

Ce document a été généré automatiquement le 19 avril 2019.

(c) Tous droits réservés 


\section{Du théâtre à la bande dessinée}

Adapter une farce médiévale en BD au collège : quels enjeux et quelle démarche?

From theater to comic strip. Adaptation of a medieval farce for the comic book in middle school: issues and process

Armelle Hesse-Weber

1 Le présent article a pour but d'analyser et de présenter les enjeux d'une expérience d'enseignement menée dans une classe de cinquième de collège, expérience facilement transposable à un autre niveau, les exigences en termes d'écriture, de production d'images et les aides apportées aux élèves pouvant tout à fait être adaptées aux compétences, savoirs et savoirs faire des élèves ainsi qu'aux exigences institutionnelles des quatre années de collège.

2 Elle permet de combiner la lecture/compréhension d'un texte littéraire du Moyen Âge $L a$ Farce de Maître Pathelin (2010), farce anonyme du XVe siècle, la production écrite, le travail sur la langue et sur l'image fixe, à savoir la bande dessinée (BD) et ses caractéristiques, en réception comme en production.

\section{Adapter une œuvre théâtrale en BD : les enjeux}

\section{Pourquoi adapter?}

3 L'adaptation en ce qu'elle désigne un processus de transformation d'une œuvre dans une autre sémiologie pour la rendre apte à un nouveau média et à un nouveau public est une pratique très courante dans la création artistique et littéraire et qui a évolué au cours des siècles. Désignant essentiellement le passage d'une œuvre romanesque au théâtre, sa pratique touche aujourd'hui de nouveaux supports médiatiques tels que le cinéma, la BD ou encore le clip ou les jeux vidéo. L'objectif de cet article est de montrer, d'un point de vue didactique, les intérêts multiples de l'adaptation au collège, et en particulier lorsqu'il s'agit d'adapter une œuvre théâtrale du Moyen Âge, La Farce de Maître Patelin, en BD. 
4 L'adaptation, qu'on la nomme, selon les théoriciens (Petitjean \& Hesse-Weber, 2011), transécriture, hybridation, reprise ou réécriture, est une réalité historique (Hesse-Weber, 2012) et culturelle qui s'est manifestée depuis l'antiquité jusqu'à nos jours sous les formes et avec les enjeux les plus divers. Très présente dans le champ de l'école, elle touche essentiellement les textes au programme qui sont adaptés à l'âge des élèves en conservant la même sémiologie (on peut citer par exemple les adaptations homogénériques de la collection L'école des Loisirs qui propose des versions simplifiées des œuvres patrimoniales comme L'Odyssée, Le roman de Renart, Yvain... éditions d'ailleurs conseillées par l'institution). En revanche, il existe assez peu d'adaptations transgénériques, la narration étant largement privilégiée au collège sous la forme de romans.

5 L'activité, menée en classe de cinquième, s'inscrit dans les programmes officiels de 2008', mais aussi de $2016^{2}$, avec la volonté de mettre en place une activité de «transécriture » (Gaudreault \& Groensteen, 1998) qui permette une véritable appropriation du texte source support de la réécriture et d'en vérifier la compréhension en lecture. Elle permet de montrer que lire c'est interpréter et qu'adapter c'est faire des choix, comprendre, sélectionner et ainsi mémoriser les éléments jugés importants du texte source.

6 J'ai montré que l'adaptation théâtrale est une opération de lecture-écriture-réécriture qui obéit aux mêmes opérations que celles décrites dans les modèles rédactionnels (GarciaDebanc, 1986 ; Bessonnat, 2000) à savoir la planification, la mise en texte et la révision, et que sa pratique pouvait donc jouer un rôle dans l'apprentissage de l'écriture et de la lecture en ce qu'elle est un axe de lecture en même temps que de création d'une nouvelle œuvre autonome (Hesse-Weber, 2010). Ces opérations observées dans les textes déjà adaptés peuvent aider les apprentis scripteurs que sont les collégiens à améliorer, dans une démarche consciente, leurs propres écrits. L'adaptation fait ainsi prendre conscience du fait que l'écrivain se nourrit de ses propres lectures, progresse en réécrivant son texte ou celui des autres, à l'encontre de l'idéologie du don et de l'inspiration. Elle permet également d'aborder de manière concrète la notion de genre et d'engager une réflexion sur les phénomènes d'intertextualité et de littérarité qui sont des éléments clés des programmes du second degré. En tant que transformation d'une œuvre d'une sémiologie dans une autre, l'adaptation est un bon moyen d'appréhender la notion de généricité, rend les élèves sensibles au phénomène d'interpénétration des genres et incite à relever les variations entre les œuvres. Elle pose la question de la fidélité au texte source en même temps qu'elle permet d'aborder la notion de création. En écriture, transformer un texte « déjà-là » permet aussi aux élèves d'éviter le syndrome de la page blanche ou de la panne d'inspiration qui peuvent générer des blocages auprès des plus fragiles.

\section{Pourquoi adapter un texte de théâtre?}

7 Le théâtre a longtemps été oublié des manuels scolaires du collège où il a souvent été prétexte à des activités ludiques de mise en voix ou encore de lecture à plusieurs voix, avec, érigés en modèles, les textes de théâtre du XVII ${ }^{\mathrm{e}}$ siècle qui pouvaient faire obstacle à la lecture et à l'écriture du théâtre. Les choses ont bien évolué ces deux dernières décennies grâce à la prise en compte des théories de références portant notamment sur le discours, la communication et la théâtralisation. L'enseignement du théâtre a largement 
bénéficié des recherches en sémiologie du théâtre et il se concrétise aujourd'hui sous des formes différentes :

- comme partie intégrante de l'enseignement du français ;

- comme pratique artistique et culturelle en milieu scolaire ;

- comme discipline artistique dans le cadre des enseignements de spécialité au lycée où des progrès ont été entrepris grâce au partenariat entre éducation/culture et professionnels du théâtre, donnant lieu à un enseignement structuré et validé au baccalauréat ;

- avec une ouverture sur les textes contemporains, notamment au lycée...

8 Les programmes de 2008 traduisent un réel intérêt de l'éducation nationale pour l'éducation artistique et culturelle (relance des classes à PAC, extension des classes à horaires aménagés et pratique artistique et culturelle dans le cadre de l'accompagnement éducatif, même si elle ne constitue en 2008 que 19 \% des activités). Il s'agit de concilier la création artistique, les valeurs éducatives du théatre et l'expression dramatique, contre l'idée selon laquelle le théâtre ne s'enseigne pas, et contre une instrumentalisation de ce dernier par l'école, comme simple outil d'intégration ou de motivation.

9 Le théâtre comme pratique artistique sert aussi à apprendre par le corps (situation d'apprentissage qui diffère des habitudes), à donner envie de voir du théâtre, à apprendre la complexité des langues et du monde, à mettre en voix pour faire jaillir le sens...

10 Néanmoins, il faut rester vigilants car les nouveaux programmes applicables à la rentrée 2016 pour les cycles 3 et 4 ne font plus apparaitre la notion de représentation et laissent la liberté aux enseignants d'aborder les entrées thématiques imposées, sans jamais passer par le texte dramatique s'ils en font le choix. Décision bien regrettable car la spécificité du théâtre est d'associer le texte et sa représentation.

\section{Pourquoi choisir le théâtre comme genre d'arrivée?}

11 L'activité de transmodalisation que nous proposons devrait permettre d'aborder le théâtre sous un angle qui a l'avantage de prendre appui sur la représentation, qui est l'essence même du théâtre et qui, bien souvent, est oubliée au profit du texte. En effet, le travail du scénariste de BD offre des similitudes avec celui du metteur en scène en ce qu'ils sont tous deux amenés à effectuer des choix dans le positionnement des personnages, les déplacements, mimiques des personnages et le cadrage des tableaux. Ainsi, certaines didascalies pourront être directement traduites en images, alors que d'autres gestes, mimiques, costumes ou déplacements devront faire l'objet de discussions et de choix qui donneront son caractère à l'adaptation. Adapter une pièce de théâtre en $\mathrm{BD}$, c'est passer d'un art visuel à un autre, d'une œuvre à représenter sur scène à une œuvre à représenter par le dessin et une histoire dont la mise en scène peut aider le passage à la forme verbo-iconique. L'activité oblige également les élèves à se poser des questions de mises en scène proches de celles qui sont posées lors du montage d'une pièce de théâtre.

Le théâtre peut aussi apparaitre comme un genre facile à adapter en BD en raison de la présence de paroles rapportées au discours direct qui peuvent être totalement ou partiellement reprises. En outre, le découpage en scènes et en actes ou en tableaux peut faciliter la saisie de la trame narrative et ainsi, la transposition en planches et l'intégration de l'activité dans le format de la séquence didactique. 
13 Tout comme le conte, la fable ou le poème, la pièce de théâtre se prête aussi plus facilement à l'adaptation en BD lorsqu'on la veut fidèle à l'œuvre originale, en raison de sa brièveté, et du format limité en volume du média d'arrivée (48 pages en moyenne pour une $\mathrm{BD}$ « classique »).

La transformation en BD peut aussi avoir pour vocation de rendre plus attractif le genre dramatique, souvent considéré comme classique, voire inabordable, en raison des textes enseignés dans le secondaire, dont les auteurs restent majoritairement ceux du XVII siècle avec une prééminence des comédies de Molière, qui, même si elles font encore rire le public, offrent de plus en plus de résistance aux élèves de collège en termes de vocabulaire et d'univers de référence. Transformer le théâtre dans un média réputé plus moderne permet, tout en s'appropriant le texte et le genre de départ, de lui donner un deuxième souffle dans une activité qui peut paraitre à première vue ludique aux élèves, même si on sait que cette vision est erronée, la maitrise des codes des deux genres et du rapport texte/représentation et texte/image induisant une difficulté supplémentaire.

Enfin, lire le théâtre en BD permettra aux élèves, à travers la lecture cursive ou accompagnée de ces œuvres foisonnantes, d'élargir leur connaissance des textes de théâtre, mais aussi de s'ouvrir à d'autres genres de BD que ceux vers lesquels ils sont le plus attirés. Ces lectures leur permettront bien sûr également de mieux appréhender le travail d'adaptation qui va leur être demandé.

\section{Essor de la BD et des transpositions en mode verbo- iconique}

\section{Un marché en pleine croissance}

Le marché de la BD est couramment divisé en quatre genres : franco-belge, manga, comics et roman graphique. Tous genres confondus, le nombre de publications annuel a presque quadruplé depuis quinze ans, passant de 1137 nouveautés en 2000 à 3946 en 2014. Depuis près de trente ans, on constate en France une très forte croissance des productions et ventes de BD. En effet, 39 millions d'albums ont été vendus en 2015 pour un chiffre d'affaires de 459 millions d'euros ( $+10,6 \%$ en volume et en valeur $)^{3}$. La sortie des derniers opus des aventures d'Astérix (Ferry \& Conrad, 2015), et de Titeuf (Zep, 2015) ${ }^{4}$ n'est pas étrangère à cette croissance puisqu'elle contribue à peu près à $3,6 \%$ de ces résultats, néanmoins, sans cela, le marché aurait tout de même connu une croissance de $7 \%$ en chiffre d'affaires.

17 Selon les données des dossiers annuels Livres Hebdo/Ipsos, le genre représente désormais $10 \%$ des ventes de livres, or, il se trouve que ce sont les jeunes adolescents qui en sont les principaux consommateurs. Selon une étude réalisée auprès d'un échantillon de 1500 jeunes âgés de 7 à 19 ans entre le 25 mai et le $1^{\text {er }}$ juin $2016^{5}$, le livre préféré des filles et des garçons dans toutes les tranches d'âge interrogées est Harry Potter. Mais viennent immédiatement après dans le palmarès, les deux BD sorties en 2015 et déjà citées que l'on retrouve également parmi celles qui sont les plus empruntées dans le CDI de mon établissement scolaire, à savoir Titeuf et Astérix. Par ailleurs, les champs d'investigation de la BD n'ont cessé de se diversifier et tous les genres sont aujourd'hui représentés (historique, psychologique, fantastique, science-fiction l'autobiographique...). 


\section{La tentation des classiques} programmes télévisés ou de compétitions sportives, les transformations de textes littéraires classiques en mode verbo-iconique représentent une nouvelle tendance. Des collections spécialisées ont été créées par certaines maisons d'édition comme la collection Romans de Toujours des éditions Adonis reprise par Glénat puis les éditions France Loisirs sous le titre Les Incontournables de la littérature en $B D^{6}$. Elles affichent, par cette démarche, leur volonté de « diffuser sous une forme agréable et moderne les Trésors de la littérature romanesque mondiale pour qu'ils soient accessibles au plus grand nombre. [...] La qualité du scénario, qui respecte au plus près le style même de l'auteur, garde intacte la force de l'œuvre originale. [...] Pour le grand public, ces BD seront aussi une belle incitation à (re)lire l'œuvre originale ${ }^{7} »$.

C'est aussi l'ambition des éditions de Sèvres creées par L'école des loisirs, pilier de l'édition jeunesse qui consacre une partie de sa collection, aux côtés d'auteurs de BD reconnus comme Zep (l'auteur de Titeuf a inauguré la collection dans un registre inattendu en septembre 2013 avec la publication d'Une histoire d'hommes), à des adaptations de classiques de la littérature ou de textes de qualité et appartenant à son catalogue d'origine ${ }^{8}$. Si le théâtre est moins adapté que les romans classiques, on trouve néanmoins Petit à Petit qui publie des BD, de la littérature jeunesse, de la littérature et des beaux livres et consacre une partie de son catalogue de BD à des opus destinés aux adultes, à une série historique mais aussi à de nombreuses adaptations: "Chanson en $\mathrm{BD}$ », « Légende en $\mathrm{BD}$ », «Littérature en $\mathrm{BD}$ » et « Théâtre en BD ». Dans cette dernière catégorie, apparaissent des adaptations des grands classiques du théâtre (Le Cid, Phèdre et Cyrano de Bergerac parus en 2006, Ubu Roi en 2009...). Les éditions Delcourt consacrent également une partie de leur catalogue à des adaptations théâtrales de Molière notamment avec Le Tartuffe en 3 volumes publiés entre 2008 et 2010 et Dom Juan publié en 2010.

En adaptant plutôt des œuvres classiques étudiées au collège, les auteurs et les éditeurs s'assurent, tout d'abord un lectorat sans prendre trop de risque, puis réconcilient ce dernier avec le théâtre, avec peu d'investissement en termes de transformation. En effet, la plupart du temps, les textes des auteurs sont respectés à la lettre et seules quelques onomatopées sont ajoutées ou encore des effets de jeu avec la police de caractère viennent appuyer le texte derrière lequel les bédéistes se retranchent avec le plus grand respect et la plus grande discrétion.

21 Si ce sont les pièces classiques qui sont les plus adaptées, notons tout de même l'audace des éditions des Arènes. L'éditeur à succès d'E. Joly, de D. Robert ou de V. Trierweiler ( Merci pour ce moment) s'est en effet lancé en 2015 dans la BD et publie une pièce contemporaine: Le porteur d'histoire, adaptée de la pièce de théâtre éponyme d'A. Michalik, récompensée par deux Molières en 2014 (meilleur auteur, meilleur metteur en scène).

Enfin, d'autres éditeurs sont allés jusqu'à se spécialiser dans l'adaptation de pièces de théâtre en $\mathrm{BD}$, comme les éditions Vent d'Ouest qui ont transposé de nombreuses pièces de Molière (Les précieuses ridicules, Le Médecin malgré lui, George Dandin, Les Fourberies de Scapin, L'Avare).

Pratiques, 175-176 | 2017 


\section{Place de la BD au collège}

La diversification de l'offre de publication, le succès auprès des jeunes lecteurs et en creux la motivation qu'elle permet d'inscrire dans l'activité proposée à la classe ainsi que sa proximité avec le genre source, le théâtre, font de la BD un genre idéal à explorer au collège.

Loin de la vision d'une œuvre simplifiée voire simpliste et d'une lecture mineure et déconsidérée, la transformation dans ce nouveau genre implique, outre une prise de distance avec le texte source, la maitrise des codes de signification qui lui sont propres. Mais alors que le succès de la BD ne cesse de croitre et qu'elle a enfin été légitimée par l'institution scolaire, notamment dans les programmes de 1995 où elle était étudiée en relation avec différents discours et où sa nature verbo-iconique était soulignée, on peut déplorer avec B. Bomel-Rainelli et A. Demarco (2011, p. 81-92) l'abandon, dans les textes officiels de 2008, de toute référence à un corpus ${ }^{9}$ et la disparition de la notion de BD dans les programmes pour le français. Elle s'y trouve réduite sous le terme générique d'image ou alors à sa fonction narrative, sans que l'accent soit mis sur sa double nature :

Il est dommage qu'une lecture littéraliste des programmes pousse les éditeurs scolaires à diminuer mécaniquement la place de la $\mathrm{BD}$, son étude, et cela quelquefois sans donner aux autres formes d'image une place autre que décorative. Ce revirement brusque dû aux programmes officiels de 2008 parait paradoxal au moment où cet art complexe manifeste une formidable vitalité artistique (explosion formelle, concerts dessinés, BD numérique...) et économique (4 863 titres publiés en français en 2009). Cette interprétation restrictive prive enseignants et élèves seul type de BD lu par les adolescents, alors que la BD tend, peut-être plus que les autres, des passerelles vers d'autres formes d'art, la littérature verbale, l'architecture, le cinéma, l'animation et les jeux vidéo. (ibid., p. 81)

Le résultat de ce changement de prescription se fait sentir dans les manuels scolaires analysés par les deux spécialistes qui notent que la disparition d'une liste de référence officielle pour la BD fait s'effondrer le pourcentage des œuvres puisées dans les listes de $1995^{10}$. L'autre conséquence de cette réforme est qu'après 2008, l'examen des titres proposés par les manuels scolaires fait apparaitre une restriction de l'originalité et de la nouveauté formelle: les œuvres longues sont délaissées au profit d'œuvres issues de genres courts, souvent instrumentalisés au service de la littérature de patrimoine par le biais d'adaptations destinées à faciliter la lecture de l'hypotexte plus qu'à permettre une analyse générique comparative et à faire émerger les spécificités de l'hypertexte.

Ainsi, faire adapter le théâtre en BD au collège, c'est prendre en considération le statut artistique spécifique de deux genres littéraires parfois mal considérés par les programmes scolaires et souvent oubliés dans la formation continue des enseignants. Cela est d'autant plus regrettable qu'ils possèdent un réel capital de sympathie auprès des élèves et répondent parfaitement à la demande qui est faite de leur constituer un parcours artistique et culturel, que ce soit par le biais de l'histoire des arts (programmes de 2008) ou du nouveau PEAC (programmes de 2016). Mais au-delà de ces éléments, l'étude de la BD permet d'aborder plusieurs aspects du programme de français que développe L. Guyon (2006) dans son article intitulé «Trois (bonnes) raisons d'étudier l'adaptation BD d'une œuvre littéraire » dans le dossier de la revue Lire au collège consacré à l'adaptation. Ainsi, outre l'incitation à connaitre le texte source, pour ce spécialiste de la 
pédagogie de la $\mathrm{BD}$, étudier la $\mathrm{BD}$ pour elle-même et dans son rapport avec le texte dont elle est l'adaptation permet d'aborder ces trois éléments essentiels :

- la notion de genre avec les spécificités de chacun : transcrire l'action, décrire, représenter un personnage...

- les grandes règles du récit : auteur, narrateur, marqueurs spatiaux-temporels, organisation du récit...

- les notions d'intertextualité et de conditions de production de l'œuvre : attentes de lecture, adaptation et illustration, représentation de l'irreprésentable, rapports d'intertextualité, conditions de production...

- enfin, cette opération de transposition d'un genre dans un autre va favoriser le travail en projet en faisant dialoguer les disciplines.

\section{Adaptation transgénérique au collège : l'exemple de $L a$ Farce de Maître Pathelin adaptée dans le mode de la BD}

\section{Un travail en atelier et en projet pluridisciplinaire}

Il s'agit d'inscrire cette activité d'écriture longue dans une pratique sociale, dans laquelle chaque élève sera investi d'un but concret et conscientisé, à même de lui permettre de s'engager dans les tâches. Dans cette perspective, nous avons tenté de respecter les caractéristiques de cette conception praxéologique de l'écriture en projet, reprises en ces termes par C. Masseron (2008, p. 83) :

Rappelons qu'il s'agit de conduire les élèves à la formulation d'un projet de travail collectif et complexe, étalé dans le temps, négocié au fil de son avancement lors des séances de conseil, co-évalué (la table de lecture des textes produits) et finalement diffusé auprès d'un public élargi qui ne soit pas les seuls membres de la classe concernée par le projet.

Cette conception dynamique et motivante de l'écriture en projet donne du sens aux apprentissages en liant les savoirs à une situation problématique à résoudre, dont les obstacles et les enjeux devront être soigneusement délimités et identifiés. Elle favorise la mise en situation des savoirs et favorise leur assimilation dans une application concrète. Lorsqu'elle prend la forme d'un atelier d'écriture, elle comprend des phases successives décrites par C. Garcia-Debanc (1989), qui doivent être clairement indiquées au scripteur :

- les phases successives d'activités et leur minutage approximatif,

- la formulation des consignes, ou énoncés des tâches à effectuer,

- la nature des inducteurs, selon les cas, textes, mots ou éventuellement objets proposés aux participants pour déclencher l'écriture,

- les modalités et matériel nécessaire pour la socialisation des écrits,

- les axes principaux d'analyses des productions.

Choisir la BD comme genre d'arrivée permet donc de donner davantage de sens aux pratiques de lecture et d'écriture dans une démarche de projet, telle qu'elle est définie par C. Garcia-Debanc (2007), à la suite de J.-F. Halté et d'A. Petitjean.

Les projets d'écriture s'appuient sur une interaction entre lecture et écriture, l'analyse de textes d'auteurs permettant de dégager des critères guidant l'écriture. L'observation des textes d'auteurs donne lieu à l'élaboration d'outils, sous la forme de grilles de critères ou de questionnaires, qui peuvent aider les élèves à relire les textes produits dans la classe, pour en repérer les réussites et les dysfonctionnements et aider à leur réécriture. Dans certains cas, la mise en œuvre 
d'« activités décrochées » en orthographe, grammaire ou conjugaison permet de construire les savoirs nécessaires pour procéder à ces réécritures. Ces activités décrochées peuvent porter sur des contenus de grammaire de phrase (la gestion des accords ou la morphologie verbale) mais aussi de grammaire de texte (l'utilisation pertinente de substituts ou le choix des temps verbaux du passé).

En ce que l'activité nécessite une collaboration avec le professeur d'arts plastiques sur le caractère verbo-iconique de la $\mathrm{BD}$, elle engage également un travail pluridisciplinaire qui favorise une conception transversale de l'écriture. Dans la mesure où le projet met en relation deux disciplines différentes, l'art plastique et le français, il implique un travail de concertation entre collègues, dont les difficultés et les intérêts ont été décrits par A. Petitjean (1981) dans un numéro de Pratiques consacré au travail en équipe, qui aura permis d'être attentif, notamment, au risque de la hiérarchisation des disciplines.

31 Notons aussi que cette séquence que nous allons décrire reste tout à fait d'actualité dans le cadre des nouveaux programmes pour le collège ${ }^{11}$, applicables à la rentrée 2016 . En effet, ces derniers préconisent l'étude de l'image en relation avec les textes, le travail en interdisciplinarité ainsi que la pédagogie de projet avec la mise en place des enseignements de pratiques interdisciplinaires (EP). Ils permettent de croiser les disciplines et doivent conduire à une production concrète, évaluée au diplôme national du brevet.

\section{Une immersion préalable dans les deux genres}

32 Le genre d'arrivée, la BD, a été choisi car il est inscrit au programme de la classe en arts plastiques et permet de faire travailler en interdisciplinarité le professeur de français et le professeur d'arts plastiques. Il ne s'agit en aucun cas ici de chercher la perfection graphique, mais d'expérimenter des choix narratifs et esthétiques facilités par l'écriture transgénérique qui permettra de se concentrer sur l'économie narrative de la BD et la relation texte/image plutôt que sur une création originale.

En ce qui concerne l'organisation du projet et sa place dans la progression annuelle, il démarre au premier trimestre après une séquence sur le fabliau, qui a permis aux élèves de revoir les caractéristiques du récit au passé et d'analyser, notamment, la parole rapportée au discours direct et son insertion dans le récit.

La séquence sur la farce permet d'approfondir le travail sur les dialogues en distinguant les répliques de théâtre des dialogues de fabliaux, leurs caractéristiques et leurs visées. Là où dans le fabliau, le dialogue sert à rendre le récit plus vivant et à révéler des informations sur les personnages par leur parlure, le niveau de langue employé..., au théâtre, il s'agit, pour l'enseignant, de montrer qu'en l'absence de narrateur et de récit introducteur, l'intrigue toute entière s'inscrit dans la parole des personnages. Le travail d'adaptation en BD permettra d'observer le lien entre le texte et l'image, cette dernière étant absente des deux autres genres, et d'établir l'importance du dessin dans l'économie de l'histoire en opérant des choix (que dire ? que montrer? dans quel but?)

Un tableau récapitulatif (Annexe 1) décline les différentes séances avec l'articulation lecture, écriture, langue dans la séquence portant sur la lecture intégrale de La Farce de Maitre Pathelin puis son adaptation en BD par les élèves.

L'expérience est complétée par une sortie scolaire au Salon du livre et de la presse jeunesse à Montreuil et notamment, par l'inscription des élèves à un atelier sur la BD (exposition intitulée $28 \mathrm{~W}$ ) avec pour support des planches de M. Bonhomme et de C. Gaultier. 
Parallèlement à l'étude de l'œuvre intégrale, la sortie au salon du livre est donc préparée en classe. La documentaliste de l'établissement vient proposer aux élèves des BD des deux auteurs qui leur seront présentés lors de l'exposition $28 \mathrm{~W}$. Afin de ne pas déflorer la découverte de l'atelier qui leur est réservé à Paris, il leur a simplement été demandé d'observer les planches et le contenu des vignettes et de donner leur avis sur les différentes manières d'approcher le dessin par les deux auteurs. Ils ont pu remarquer la noirceur des traits qui marque les œuvres de $\mathrm{C}$. Gaultier où le dessin est tout en hachures et se suffit souvent à lui-même puisque de nombreuses vignettes ne sont pas accompagnées de séquences parlées. Ils ont pu noter également l'alternance de voix-off descriptives et de séquences parlées venant succéder aux scènes muettes. Dans Robinson Crusoé (Gaultier \& Defoe, 2008), la déclinaison de l'œuvre de D. Defoe en trois volumes montre le choix de l'auteur de rester tout de même proche du texte de départ même s'il y a volonté de modernisation dans le trait. Le travail de M. Bonhomme pour Messire Guillaume (Bonhomme \& Bonneval, 2006) leur apparait moins agressif et plus poétique.

$\mathrm{Au}$ cours de la visite au salon du livre et notamment lors de l'atelier, les élèves ont pu découvrir les différentes étapes de la fabrication d'une planche de BD à travers les approches de deux auteurs aux techniques très différentes. C. Gaultier livre, par exemple, à travers des originaux de Donjon Potron-minet (Sfar, Trondheim \& Gaultier, 2008), du Fantôme de l'Opéra et des recherches sur Robinson Crusoé, deux des techniques qu'il utilise pour tracer efficacement ses aventures: le stylo et le crayon, sans jamais, ou rarement, s'essayer à des dessins préparatoires. Quant aux planches de M. Bonhomme (2005) pour Le voyage d'Esteban, elles révèlent les différentes étapes du processus de création de la BD, de la recherche aux croquis, du scénario aux dialogues, du crayon de papier à l'encrage à la plume... Notons que l'intérêt des élèves est renforcé par le fait que les planches du premier auteur constituent des adaptations d'œuvres classiques : celle de G. Leroux pour Le fantôme de l'opéra et de D. Defoe pour Robinson Crusoé et que l'activité d'adaptation qu'ils auront à effectuer leur a été annoncée. Les techniques et étapes de travail observées nourriront leur travail aussi bien avec leur professeur d'arts plastiques pour les questions de choix technique et graphique qu'avec le professeur de français pour les étapes de fabrication et le découpage narratif.

\section{Du théâtre à la BD : un jeu de lectures-réécritures pour améliorer les compétences des élèves}

En classe, l'étude intégrale de La Farce de Maître Pathelin achevée, les élèves sont à même d'en dégager les principales lignes de force. Au moment de la séance de bilan de la lecture, les élèves proposent donc un découpage de la pièce en 7 tableaux, qui constitueront autant de groupes de travail que de planches de BD à décliner en vignettes. Cette étape fondamentale permet de vérifier la compréhension de l'œuvre et de commencer à réfléchir sur les éléments essentiels à transposer. Pour y parvenir, on demande aux élèves de formaliser la démarche : ils proposent de se fonder sur les étapes importantes de l'histoire et de s'appuyer pour cela sur le schéma narratif étudié en sixième pour analyser la structure du conte et sur la structure de la pièce de théâtre (scène d'exposition, problème ou nœud de l'intrigue, actions qui conduisent au dénouement) pour en dégager les grandes parties. Un travail individuel puis une mise en commun font aussi observer que les déplacements des personnages et les changements de 
lieux structurent l'histoire. Après validation des propositions de découpage, les tableaux sont déterminés comme suit :

- Tableau 1 : scène d'exposition, dans leur maison, Pathelin, avocat désargenté se dispute avec sa femme Guillemette à propos du manque d'argent et de vêtements. Il lui promet du drap et s'en va à la foire.

- Tableau 2: à la foire, Pathelin négocie du drap avec Guillaume le drapier (flatterie, promesse, tromperie). Il en obtient à crédit mais n'a pas l'intention de payer. Invitation à récupérer l'argent autour d'une oie.

- Tableau 3 : retour de Pathelin à la maison, il raconte à sa femme sa duperie et la prépare à la scène de tromperie (théâtre dans le théâtre : elle devra faire croire qu'il est malade).

- Tableau 4 : Guillaume vient réclamer son dû chez Pathelin qui feint la folie avec l'aide de sa femme.

- Tableau 5 : Agnelet se présente chez Pathelin pour qu'il le défende au tribunal : il a tué des brebis de son maitre.

- Tableau 6: au tribunal Pathelin se retrouve en face de Guillaume, le drapier plaignant et maitre d'Agnelet qui confond les deux affaires (drap, moutons). Il est déclaré fou et Agnelet acquitté grâce au stratagème de Pathelin (répondre « bée » aux questions)

- Tableau 7 : à l'extérieur du tribunal, Agnelet refuse de payer son avocat et se moque de lui (reprise des onomatopées).

$\mathrm{Au}$ cours de la séance suivante, on propose aux élèves de faire le bilan de ce qu'ils ont pu observer, à la fois lors de la lecture des BD de C. Gaultier et de M. Bonhomme, et lors de leur visite au salon du livre. Pour mieux leur faire appréhender la notion d'adaptation, un extrait de la scène d'exposition du Médecin malgré lui de Molière ainsi que son adaptation en BD sont également proposés comme étayage en lecture comparative. Le bilan permet de s'interroger notamment sur le choix du graphisme, l'expression des personnages qui traduit les sentiments et vient soutenir l'intonation des propos, la place des personnages dans la case, leur attitude, la manière dont les dialogues sont disposés dans les bulles. Elle met aussi en évidence la représentation du temps et permet de montrer comment se matérialisent les notions d'ellipses, de pauses, ou d'accélérations. L'analyse de ces documents amène enfin les élèves à constater qu'on ne peut pas tout conserver et qu'il s'agira de faire des choix, puisqu'il a été décidé de limiter la création finale à 7 planches pour des raisons de temps.

Un premier jet d'écriture est introduit par un travail collectif en classe qui vise, à partir des observations des élèves, à donner un exemple de ce qui est attendu. Le début de la scène première de La Farce de Maitre Pathelin est donc transposé en classe avec les élèves. (Annexe 2). Placés en ilots, ils réfléchissent par groupes de quatre et une mise en commun de leurs remarques et propositions est faite en classe. Elle révèle leur difficulté à visualiser la scène qui doit être dessinée et le fait que la rareté des indications scéniques ne leur facilite pas la tâche. Ils sont alors amenés à s'interroger sur la mise en scène de la pièce qui devrait les aider à mieux se représenter les gestes, placements et déplacements des personnages. On passe donc par le jeu scénique pour faciliter la transposition verboiconique.

41 En arts plastiques, le programme de cinquième porte sur la construction d'une narration à partir d'une ou plusieurs images. Une autre entrée au programme, intitulée « L'image et son référent » amène à explorer le sens produit par la déformation, l'exagération, la distorsion et à ouvrir sur les questions de la ressemblance et de la vraisemblance, de la citation et de l'interprétation. Par le travail de transposition et d'adaptation, c'est la 
question de la ressemblance qui est posée, ici avec un référent textuel. Une activité liminaire est proposée aux élèves : ils doivent improviser autour d'une planche avec des vignettes et confronter leurs propositions. Cette introduction permet au professeur de faire le point sur ce qu'ils savent déjà sur la BD (technique de narration, vocabulaire...). Les élèves, par groupes de 3 à 4 , doivent parallèlement, en cours de français, décliner les tableaux en vignettes. Ils ont déterminé avec le professeur d'arts plastiques qu'une moyenne de 6 à 10 vignettes par planche permettait une bonne lisibilité. Ils précisent suivant le modèle du précédent tableau (Annexe 2), et pour chaque vignette, ce qui sera dessiné, en détaillant les procédés employés : les cadrages, les angles de vue, l'identité des personnages présents, les éléments du décor. Ils indiquent également le texte à faire figurer et sa forme : encadré, bulle, onomatopée...

Cette mise en forme est évaluée afin de mettre l'accent sur cette étape importante de la transposition et afin que les élèves, impatients de dessiner, ne négligent pas tout ce travail de préparation indispensable qui a précédé. Chaque groupe dispose du texte de $L a$ Farce de Maitre Pathelin et des consignes de travail matérialisées dans un tableau tiré de l'ouvrage de R. Hector (2000: 5), Petits trucs pour réaliser ta B.D.

Une grille de critères est élaborée pour faciliter la prise en compte des éléments et permettre une autoévaluation qui pourra également les conduire à une amélioration progressive de leurs travaux par la réécriture. Elle fait apparaitre les critères d'évaluation et rappelle en regard les outils à la disposition des élèves.

L'évaluation du premier jet met en évidence la difficulté des élèves à se détacher du texte d'origine et à réduire la part des dialogues de théâtre dans la BD. En recourant à d'autres exemples d'adaptations comme Molière, Le Médecin malgré lui (Molière \& Cady, 2005), il s'agit de leur montrer que la part des dialogues dans une vignette ne peut pas être trop importante si on veut laisser de la place à l'image. Ils devront donc, à la fois réduire et synthétiser les propos des personnages, et quand cela s'avère nécessaire, faire parler les images et au besoin découper les répliques les plus longues dans plusieurs vignettes. Ils seront aussi amenés à s'interroger sur ce qui est mis en scène: est-ce essentiel à l'histoire ? L'intrigue progresse-t-elle ? Pour sauvegarder la cohérence du récit, il s'agira de vérifier les raccords entre chaque case, de veiller à l'identité graphique des personnages ou des lieux, de réviser le découpage du scénario pour éviter les redondances et de modifier parfois le nombre de vignettes. Si les opérations de suppression et d'ajout sont ancrées dans les pratiques de réécriture, celle de substitution, utile pour réduire la longueur de certaines répliques, doit être davantage accompagnée. Comment dire la même chose autrement, de manière plus synthétique? On ne peut, par exemple, conserver le récit que Guillemette fait de la fable du corbeau, en raison de sa longueur, tout comme il apparait nécessaire de supprimer les passages de paroles rapportées au discours direct, insérés dans les propos de Pathelin lorsqu'il rapporte à sa femme sa conversation avec le drapier ou dans d'autres endroits du texte (scène de théâtre dans le théâtre, restitution des faits au tribunal...). Un travail sur la langue, à partir d'extraits de la pièce, est nécessaire pour accompagner les élèves dans cette transformation. On observe à cette occasion leur difficulté à se détacher du texte source en raison de leur crainte de le dénaturer et de leur difficulté à conserver un registre de langue identique entre leur production et l'emprunt au texte source. Les remaniements s'effectuent avec l'aide des enseignants et après discussions entre pairs, en ilots.

Compte tenu de ces corrections apportées en classe et des outils construits au fur et à mesure dans les deux disciplines, les élèves doivent réécrire et améliorer leurs 
productions. Certains d'entre eux, à l'aise en dessin ou qui ont besoin de passer plus rapidement à des éléments concrets, choisissent de proposer des croquis rapides en regard du texte, dont ils maitrisent du coup mieux la longueur.

Un travail au CDI permet également de faire des recherches sur les personnages, leur allure, leur physique, afin de réfléchir à un modèle commun à présenter au professeur d'arts plastiques. On propose notamment aux élèves des modèles comme le fac similé de l'édition de Pierre Levet ${ }^{12}$ (1489), des premières de couverture de différents éditeurs de la pièce (L'École des Loisirs, éditions Babel...) et des recherches documentaires sur le costume au Moyen Âge. Avec le professeur d'arts plastiques, les élèves sont encouragés à s'émanciper des modèles pour proposer des versions plus personnelles mais qui soient en accord avec les impératifs du texte (les robes portées par Pathelin et Guillemette). Plusieurs ébauches (Annexe 3) sont réalisées au cours desquelles il s'agit de se mettre d'accord sur la manière unique dont les personnages seront représentés pour maintenir une unité dans la narration. Une représentation plus moderne est proposée par un groupe (Pathelin portant jeans et baskets). Elle est discutée puis finalement abandonnée dans la mesure où ce choix impose de modifier le contenu des bulles, de moderniser les paroles des personnages et de changer le contenu de certaines répliques (celles qui évoquent les robes des personnages notamment) afin qu'elles soient en adéquation avec les images. De peur de s'éloigner trop du texte source et d'en perdre le fil, les élèves préfèrent s'en tenir à une version plus fidèle au texte source.

En arts plastiques le travail se poursuit sur les caractéristiques techniques de la BD. Les élèves sont amenés à s'interroger sur la construction cohérente d'une image, notamment dans le projet qui nous intéresse, sur l'utilisation d'un cadrage adéquat. Un document de synthèse est constitué. Il reprend les principaux plans, leur description et la manière dont on peut en interpréter le choix et l'utilisation. À partir du premier jet et de la colonne de gauche du tableau utilisé en français, le professeur fait transférer concrètement les notions de plan et de cadrage, notions qui aident également les élèves à retravailler le contenu de leur vignette et à répartir de manière plus cohérente le texte des bulles. L'expression des visages est revue pour donner davantage d'expressivité aux personnages et faire apparaitre davantage leurs traits de caractère et la valeur que les élèves souhaitent leur attribuer dans leur adaptation (Pathelin: filou, voleur/Guillaume: commerçant aisé, à l'air sévère) ou leur relation à leur profession (le juge porte une perruque, Agnelet ressemble à un mouton). On s'interroge également sur les moyens dont dispose la BD pour exprimer la surprise (association image, signes de ponctuation, Annexe 4). À cette occasion, une courte séance de langue permettra de revoir les principaux signes de ponctuation et leur sens. Le professeur d'arts plastiques, de son côté, montrera le jeu possible avec la taille, la police de caractère des lettres et des signes à partir d'exemples concrets, pour montrer comment elles peuvent apporter du sens. Le dernier jet est, à chaque demande, validé par le professeur de français qui s'assure de sa conformité avec l'histoire source et de sa correction du point de vue de la langue.

L'état final passe par un crayonné. La BD est exécutée au propre sur du papier à dessin (Annexe 5). Cette technique est privilégiée par rapport aux outils numériques pour rester proche des modèles observés en atelier au salon du livre, mais aussi parce que l'établissement ne dispose pas d'ordinateurs en nombre suffisant pour expérimenter cette pratique. Enfin, le travail de mise en couleur (feutres ou crayons de couleurs) étant à poursuivre à la maison, pour des raisons de temps, ce choix permet de ne pas être gêné par l'hétérogénéité dans la maitrise et l'équipement de l'outil numérique. Les planches 
définitives (Annexe 6) seront évaluées en français pour le récit, la cohérence des dialogues et le rapport texte/image, quant à la partie graphique et technique avec le choix des plans, du cadrage... sera évaluée en arts plastiques. La version finale sera numérisée et déposée sur l'environnement numérique de travail de l'établissement (ENT PLACE) puis reliée pour en faire un album qui sera présenté lors des portes ouvertes du collège.

\section{Conclusion}

51 L'adaptation implique des opérations telles que la révision d'un texte source (le sien au celui d'un autre) qui elle-même s'accompagne de gestes (suppression, ajout, substitution, déplacement) inhérents à l'acte d'écriture et de réécriture. Selon les époques, les buts assignés à l'adaptation ont été variables: s'adapter au contexte culturel et au public, changer de sémiologie, dépoussiérer les classiques, renouveler le genre, etc. Cette pratique a longtemps privilégié les transformations de genres narratifs (contes et romans) en mode dramatique avant de gagner de nombreux médias (film, jeux vidéo, clips...). Sur la scène scolaire, l'adaptation offre des bénéfices non négligeables pour l'acquisition de compétences en langue, en lecture, en écriture et à l'oral. En production comme en réception, elle implique un travail de lecture/réécriture qui permet d'aborder les problèmes de transformations génériques et de transmission de valeurs. Passer d'un genre à l'autre nécessite pour les élèves la compréhension en lecture du texte source et favorise son appropriation et sa mémorisation par sa réécriture. Cette activité permet d'assimiler de manière concrète les propriétés linguistiques et formelles du genre de départ et du genre d'arrivée, et ainsi la construction et la mise en œuvre de savoirs explicites sur la langue. Elle nécessite de faire des choix et d'engager un dialogue métacognitif avec ses pairs dans le travail de groupe et avec le professeur afin de les justifier, facilitant le passage de procédures (connaissances en acte) au savoir déclaratif.

Sur un plan plus pédagogique, l'adaptation d'un texte dramatique en BD donne l'occasion de faire travailler les élèves en atelier et en projet, dans lequel la pluridisciplinarité donne du sens aux enseignements par le dialogue qui est instauré entre les disciplines et par la production finale qui résulte de l'ensemble de la démarche. La diffusion de cette production permettra de valoriser le travail effectué et de lui donner du sens, en même temps qu'il fera connaitre aux autres une œuvre patrimoniale dans un média à forte valeur d'attractivité pour les collégiens et qui leur donnera peut-être envie de découvrir le texte source dans sa version dramatique.

BIBLIOGRAPHIE

\section{Instructions officielles}

Bulletin officiel spécial $n^{\circ} 6$ du 28 août 2008 . 
Bulletin officiel spécial n ${ }^{\circ} 11$ du 26 novembre 2015 - Les programmes du collège à la rentrée 2016.

\section{Fuvres}

BONHOMme, M. (2005). Le voyage d'Esteban. Toulouse : Milan.

Bonhomme, M. \& BonneVal, G. de (2006). Messire Guillaume. Paris : Dupuis.

FERRY, J.-Y. \& CONRAD, D. (2015). Astérix et le Papyrus de César. Vanves : Éd. Albert-René.

GAULTIER, C. (2008). Robinson Crusoé. Paris : Delcourt.

La Farce de Maître Pathelin (2010). Version en français moderne de F. Rachmuhl. Paris : Hatier.

MOLIÈRE \& CADY, V. (2005). Le Médecin malgré lui. Issy-les-Moulineaux : Vents d'Ouest.

SFAR, J., TRONDheim, L. \& GAUltier, C. (2008). Donjon Potron-minet. Paris : Delcourt.

ZEP (2015). Bienvenue en adolescence. Grenoble : Glénat.

\section{Textes théoriques}

BESSonnat, D. (2000). « Deux ou trois choses que je sais de la réécriture ». Pratiques 105-106, p. 5-18.

BOMEL-RAINELLI, B. \& DEMARCO, A. (2011). « La BD au collège depuis 1995 : entre instrumentalisation et reconnaissance d'un art ». Le français aujourd'hui 1, 172, p. 81-92. En ligne : www.cairn.info/ revue-le-francais-aujourd-hui-2011-1-page-81.htm.

GARCIA-DEBANC, C. (1986). «Intérêts des modèles du processus rédactionnel pour une pédagogie de l'écriture ». Pratiques 49, p. 23-49.

GARCIA-DEBANC, C. (1989). « De l'usage d'ateliers d'écriture en formation d'enseignants de français ». Pratiques 61, p. 29-56.

GARCIA-DEBANC, C. (2007). « Place et fonctions de l'écriture au collège : développer un regard d'artisan ». Écrire des textes, l'apprentissage et le plaisir. Observatoire national de la lecture, p. 57-58. gaudreault, A. \& GRoensteen, T. (dirs) (1998). La transécriture. Pour une théorie de l'adaptation: littérature, cinéma, bande dessinée, clip. Québec/Angoulême : Nota Bene/Centre national de la bande dessinée et de l'image.

GUYON, L. (2006). « Trois (bonnes) raisons d'étudier l'adaptation BD d'une œuvre littéraire ». Lire au collège 73, p. 10. En ligne : http://www.educ-revues.fr/LC/AffichageDocument.aspx? iddoc $=33484$.

HECTOR, R. (2000). Petits trucs pour réaliser ta BD. Metz : Éd. Serpenoise.

HESSE-WEBER, A. (2010). De l'adaptation théâtrale : pour une approche sémiotique et didactique. Thèse en sciences du langage : Université Paul Verlaine - Metz.

HESSE-WEBER, A. (2012). « L'adaptation théâtrale d'hier à aujourd'hui, pour une approche historique ». Dramatica 2, p. 3-22. En ligne : http://studia.ubbcluj.ro/download/pdf/734.pdf.

MASSERON, C. (2008). « Didactique de l'écriture : enseignement ou apprentissage ? », Pratiques 137-138, p. 79-96. En ligne : http://journals.openedition.org/pratiques/1153.

PETITJEAN, A. (1981). « Classe, projet, équipe : enseigner autrement », Pratiques 31, p. 33-69. 
PETITJEAN, A. \& HESSE-WEBER, A. (2011). « Pour une problématisation sémiologique de la pratique de l'adaptation ». Écho des études romanes 7, 2, p. 5-20.

RYNGAERT, J.-P. (2010). Jouer, représenter. Pratiques dramatiques et formation. Paris : A. Colin.

\section{ANNEXES}

Annexe 1

\begin{tabular}{|c|c|c|c|}
\hline \multicolumn{4}{|l|}{ En français } \\
\hline Séance 1 & Séance 2 & Séance 3 & Séance 4 \\
\hline $\begin{array}{l}\text { Lecture de l'image } \\
\text { Kermesse avec théâtre et } \\
\text { procession } \\
\text { Pieter Brueghel le Jeune } \\
\text { (Brueghel d'Enfer) et Pieter } \\
\text { Brueghel l'Ancien: forme, } \\
\text { support, technique... + } \\
\text { Les conditions de } \\
\text { représentations du théâtre } \\
\text { au Moyen Âge }\end{array}$ & $\begin{array}{l}\text { Lexique : le mot } \\
\text { «farce» } \\
\text { Étymologie, sens } \\
\text { du mot, notion de } \\
\text { polysémie. } \\
\text { Carte mentale : } \\
\text { faire le point sur } \\
\text { ce que je sais du } \\
\text { théâtre } \\
\text { Écriture : Rédigez } \\
\text { un court récit } \\
\text { dans lequel vous } \\
\text { emploierez le mot } \\
\text { «farce» avec ses } \\
\text { différents sens. }\end{array}$ & $\begin{array}{l}\text { Lecture } \\
\text { analytique de la } \\
\text { scène } 1 \\
\text { Les } \\
\text { caractéristiques } \\
\text { de la scène } \\
\text { d'exposition } \\
\text { Intertextualité : la } \\
\text { scène de ménage } \\
\text { comme scène } \\
\text { d'ouverture au } \\
\text { théâtre (ex : Le } \\
\text { Médecin malgrélui) } \\
\text { Hypothèses de } \\
\text { lecture : Imaginer } \\
\text { comment Pathelin } \\
\text { va se procurer du } \\
\text { drap sans argent }\end{array}$ & $\begin{array}{l}\text { Lecture analytique de } \\
\text { la scène } 2 \\
\text { Stratégies employées } \\
\text { par Pathelin pour } \\
\text { convaincre le drapier de } \\
\text { lui vendre le drap à } \\
\text { crédit } \\
\text { Lexique : } \\
\text { "crédit, patelin, } \\
\text { pateliner + le champ } \\
\text { lexical de la tromperie }\end{array}$ \\
\hline Séance 5 & Séance 6 & Séance 7 & Séance 8 \\
\hline $\begin{array}{l}\text { Langue : étude d'un extrait } \\
\text { de la pièce dans la langue } \\
\text { d'origine (scène 3) } \\
\text { Histoire et évolution de la } \\
\text { langue: qu'est-ce qui } \\
\text { change, qu'est-ce qui est } \\
\text { conservé dans la forme, } \\
\text { l'orthographe et la syntaxe? }\end{array}$ & $\begin{array}{l}\text { Mise en scène : À } \\
\text { la foire: la scène } \\
\text { entre Pathelin et } \\
\text { le drapier (scène } \\
\text { 2) }\end{array}$ & $\begin{array}{l}\text { Lecture } \\
\text { transversale : } \\
\text { Les indications } \\
\text { scéniques, place, } \\
\text { fonction et forme } \\
-\quad \text { relevé } \quad \text { et } \\
\text { classement (les } \\
\text { modes participe et } \\
\text { gérondif dans les } \\
\text { didascalies) }\end{array}$ & $\begin{array}{l}\text { Devoir d'écriture: } \\
\text { Rédiger une scène de } \\
\text { théâtre dans laquelle } \\
\text { vous cherchez à } \\
\text { convaincre vos parents } \\
\text { de vous laisser sortir. } \\
\text { Vous emploierez les } \\
\text { mêmes procédés que } \\
\text { Pathelin (flatterie, } \\
\text { promesse, tromperie) + } \\
\text { didascalies de formes et } \\
\text { fonctions variées }\end{array}$ \\
\hline
\end{tabular}




\begin{tabular}{|c|c|c|c|}
\hline Séance 9 & Séance 10 & Séance 11 & Séance 12 \\
\hline $\begin{array}{l}\text { Langue : les types de } \\
\text { phrases (rappel) et } \\
\text { l'emphase }+ \text { les formes de } \\
\text { phrases (affirmative/ } \\
\text { négative) et les interjections }\end{array}$ & $\begin{array}{l}\text { Lecture } \\
\text { analytique de la } \\
\text { scène } 5: \text { Pathelin } \\
\text { feint la folie: } \\
\text { scène de théâtre } \\
\text { dans le théâtre, } \\
\text { complicité avec le } \\
\text { public et comique }\end{array}$ & $\begin{array}{l}\text { Lecture } \\
\text { transversale : } \\
\text { Comique de } \\
\text { mœurs et actualité } \\
\text { de la pièce. } \\
\text { Les apartés et leur } \\
\text { rôle dans la pièce } \\
\text { (tentative de mise } \\
\text { en scène) } \\
\text { Lecture cursive } \\
\text { des scènes } 6 \text { et } 7\end{array}$ & $\begin{array}{l}\text { Contrôle de lecture } \\
\text { intermédiaire } \\
\text { Scènes } 7: \text { lieu, } \\
\text { personnages, situation } \\
\text { Comment Agnelet } \\
\begin{array}{l}\text { convainc Pathelin de le } \\
\text { défendre, préparation } \\
\text { de la scène du tribunal }\end{array}\end{array}$ \\
\hline Séance 13 & Séance 14 & Séance 15 & Séance 16 \\
\hline $\begin{array}{l}\text { Lecture analytique de la } \\
\text { scène } 8: \text { Confusion du } \\
\text { drapier au tribunal } \\
\text { Les différents types de } \\
\text { comique (comique de farce) } \\
\text { Champ lexical de la justice } \\
\text { La satire: aspects de la } \\
\text { société médiévale mis en } \\
\text { cause. }\end{array}$ & $\begin{array}{l}\text { Correction du } \\
\text { devoir d'écriture } \\
\text { Oralisation et } \\
\text { mise en scène de } \\
\text { quelques devoirs } \\
\text { avec ajout de } \\
\text { didascalies (leur } \\
\text { rôle dans la } \\
\text { scène). }\end{array}$ & $\begin{array}{l}\text { Contrôle de } \\
\text { langue : types et } \\
\text { formes de phrases, } \\
\text { interjections, } \\
\text { emphase }\end{array}$ & $\begin{array}{l}\text { Correction du contrôle } \\
\text { de lecture (scène } 7 \text { ) }\end{array}$ \\
\hline Séance 17 & Séance 18 & Séance 19 & Séance 20 \\
\hline $\begin{array}{l}\text { Correction du contrôle de } \\
\text { langue } \\
\text { Orthographe: Dictée d'un } \\
\text { extrait de la pièce }\end{array}$ & 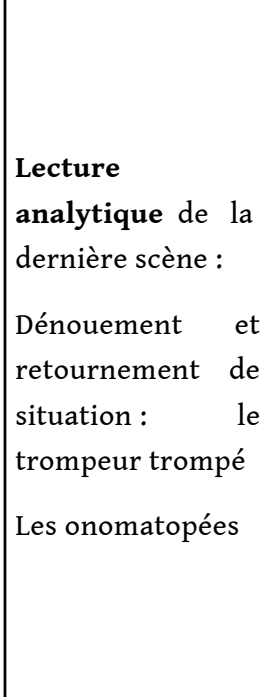 & $\begin{array}{l}\text { Bilan de la lecture } \\
\text { intégrale. } \\
\text { Caractéristiques } \\
\text { de la farce } \\
\text { Sélection r des } \\
\text { principaux } \\
\text { tableaux r à } \\
\text { conserver pour } \\
\text { l'adaptation en BD } \\
\text { (le r synopsis } \\
\text { décliné r en } \\
\text { planches) r } \\
\text { constitution des } \\
\text { groupes r }\end{array}$ & $\begin{array}{l}\text { Contrôle de lecture } \\
\text { terminal }\end{array}$ \\
\hline Séance 21 & Séance 22 & Séance 23 & Séances 24 à 30 \\
\hline
\end{tabular}




\begin{tabular}{|c|c|c|c|}
\hline 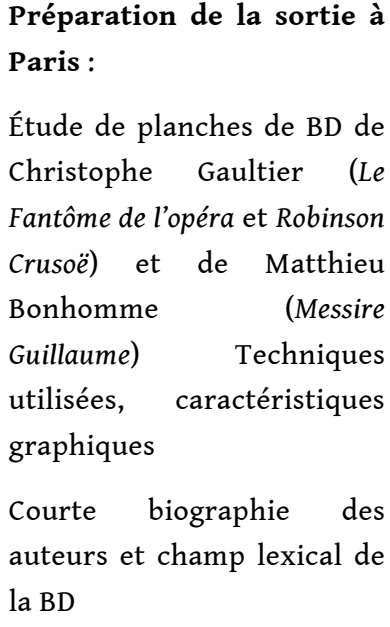 & $\begin{array}{ll}\text { Correction du } & \mathrm{du} \\
\text { contrôle de } & \text { lecture terminal }\end{array}$ & $\begin{array}{l}\text { Lecture d'un } \\
\text { extrait } \\
\text { Molière et son } \\
\text { adaptation en BD } \\
\text { Qu'est-ce qui } \\
\text { change, qu'a-t-on } \\
\text { conservé, } \\
\text { supprimé ...? }\end{array}$ & $\begin{array}{l}\text { Travail d'écriture } \\
\text { longue : } \\
\text { Adaptation de la pièce } \\
\text { en BD } \\
\text { Travail individuel et } \\
\text { par groupes } \\
\text { Alternance de séances } \\
\text { de lecture, de langue } \\
\text { et d'écriture. } \\
\text { Fin du travail à la } \\
\text { maison }\end{array}$ \\
\hline Séances menées en parallèle & En arts plastiques & & \\
\hline $\begin{array}{l}\text { Réécritures portant sur le } \\
\text { contenu des bulles } \\
\text { (sélectionner ce qui est } \\
\text { important, synthétiser, } \\
\text { substituer, ajouter, dire avec } \\
\text { les images ...) }\end{array}$ & $\begin{array}{l}\text { Étude des } \\
\text { caractéristiques } \\
\text { de la BD }\end{array}$ & La notion de plan & La notion de cadrage \\
\hline $\begin{array}{l}\text { Construction des } \\
\text { personnages (recherche de } \\
\text { documents au CDI, } \\
\text { illustrations de La Farce de } \\
\text { Maître Pathelin, proposition } \\
\text { de modernisation des } \\
\text { personnages...) }\end{array}$ & \multicolumn{3}{|c|}{ Mise en page à partir du travail effectué en français } \\
\hline
\end{tabular}

Annexe 2

\begin{tabular}{|c|c|}
\hline À gauche les indications pour le dessin & À droite, le texte des bulles \\
\hline \multicolumn{2}{|l|}{ Exemple pour démarrer la planche $\mathrm{N}^{\circ} 1$} \\
\hline $\begin{array}{l}\text { IMAGE } 1 \\
\text { Pathelin et Guillemette sont dans leur cuisine, ils } \\
\text { portent des robes déchirées, Guillemette assise est en } \\
\text { colère, Pathelin air lamentable } \\
\text { Plan d'ensemble }\end{array}$ & $\begin{array}{l}\text { TEXTE } \\
\text { Pathelin: Ma foi, Guillemette, il fut } \\
\text { un temps où j'exerçais le beau métier } \\
\text { d'avocat! }\end{array}$ \\
\hline IMAGE 2 & TEXTE \\
\hline
\end{tabular}




\section{Annexe 3}
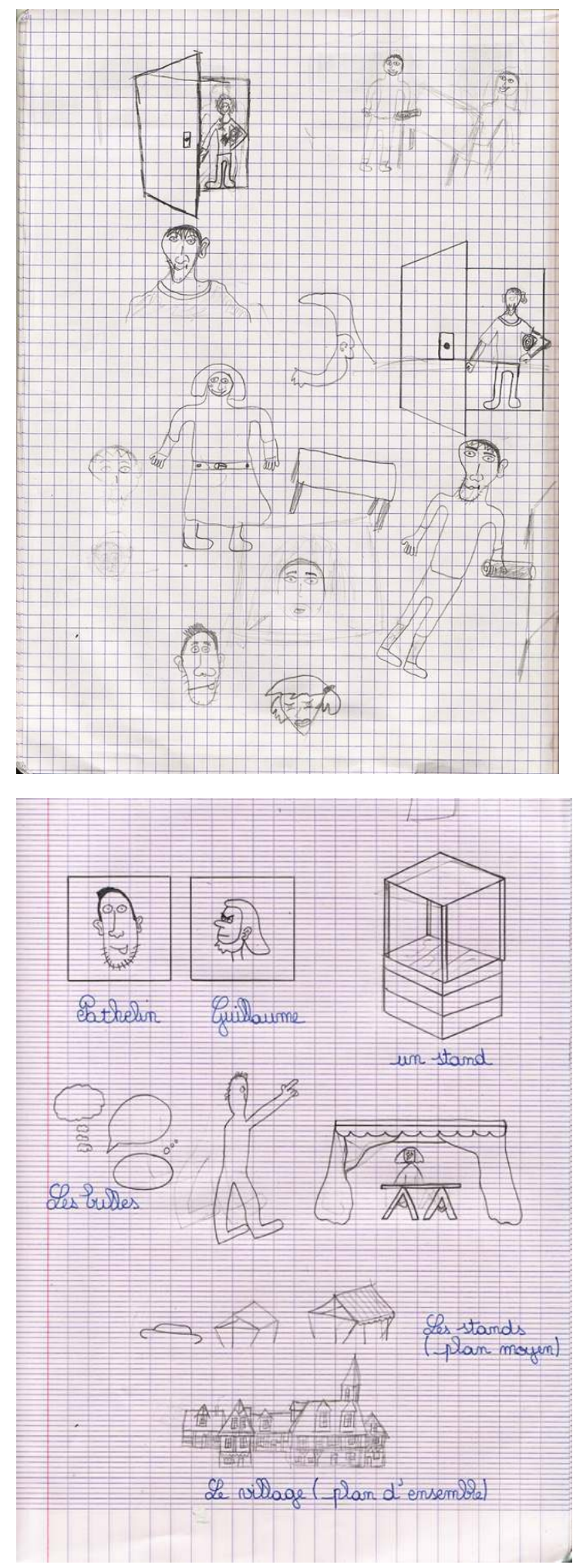

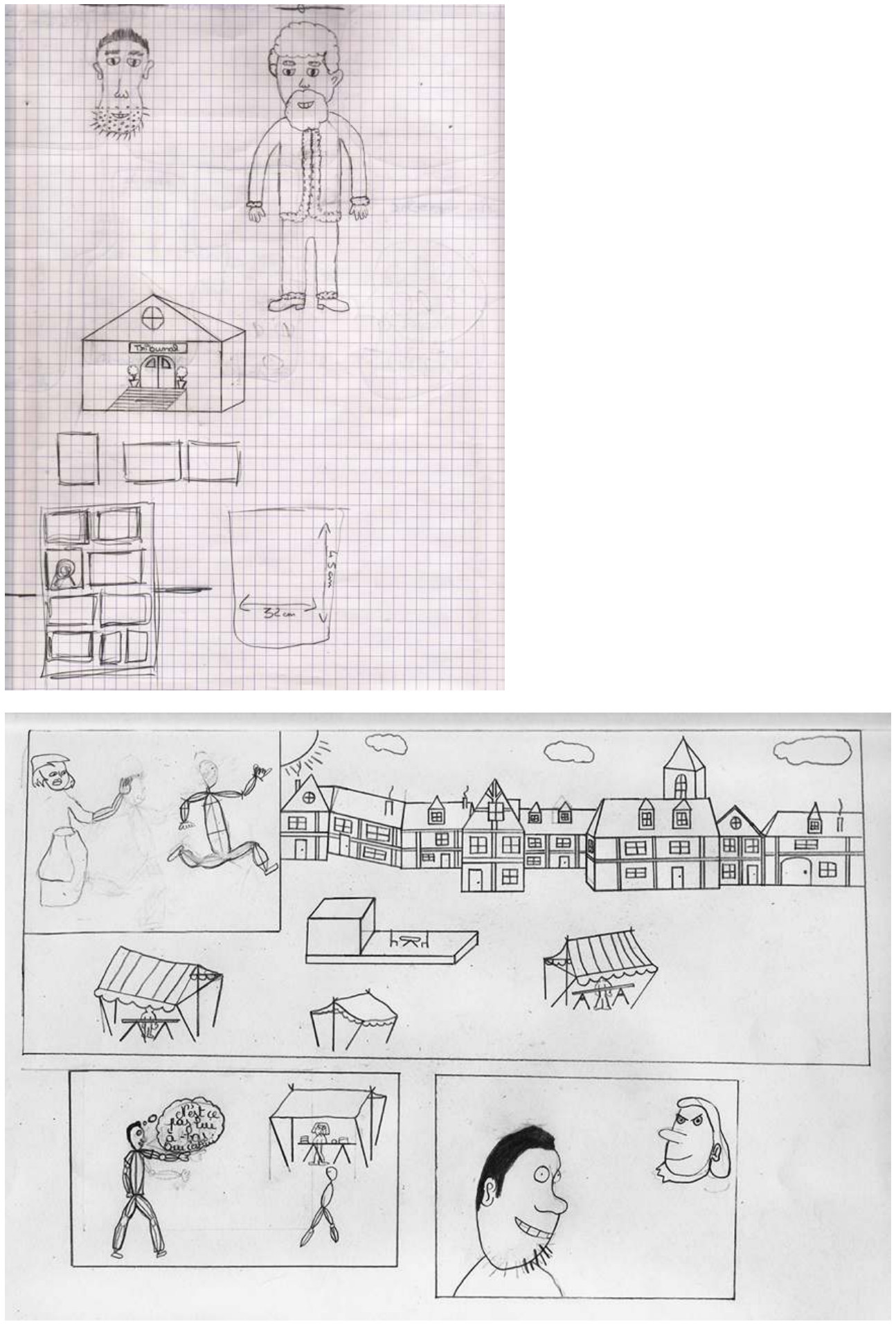

Annexe 4 


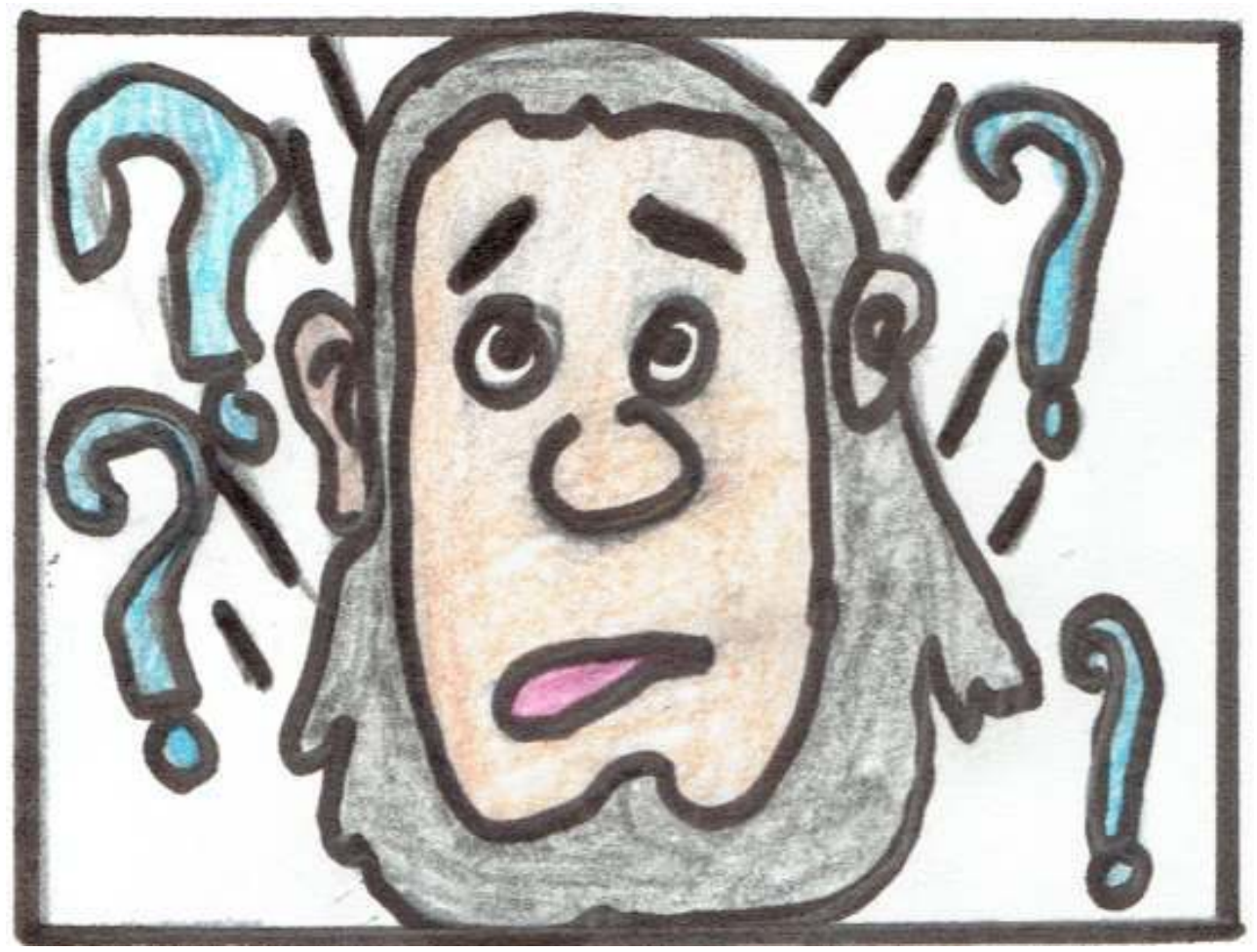

Annexe 5

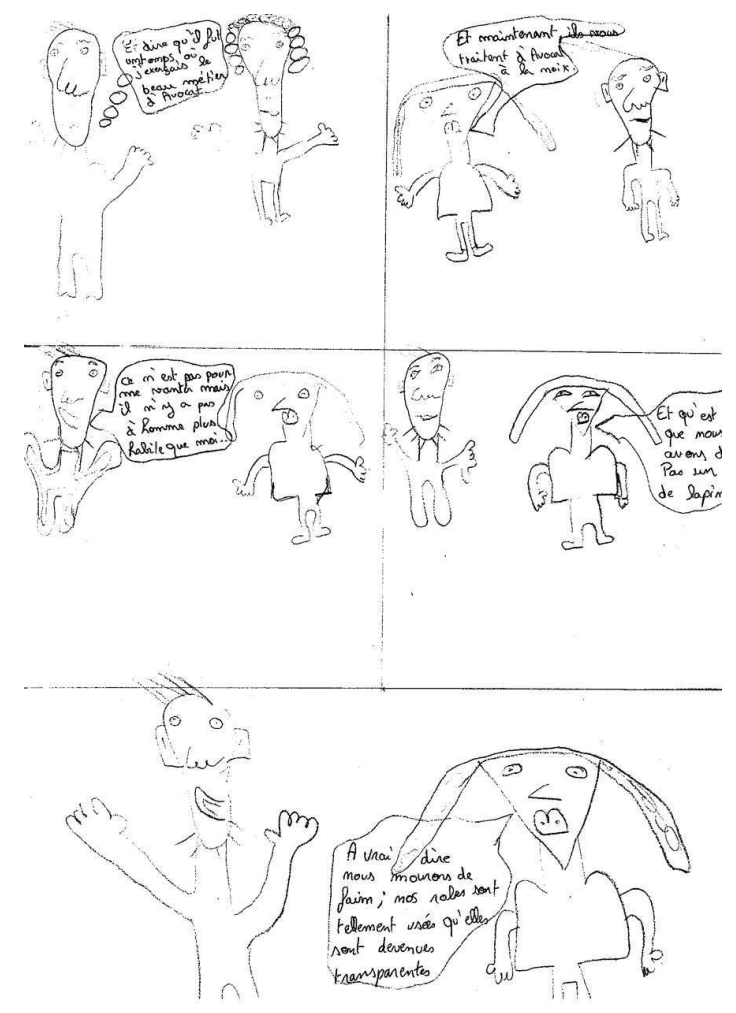

Annexe 6 

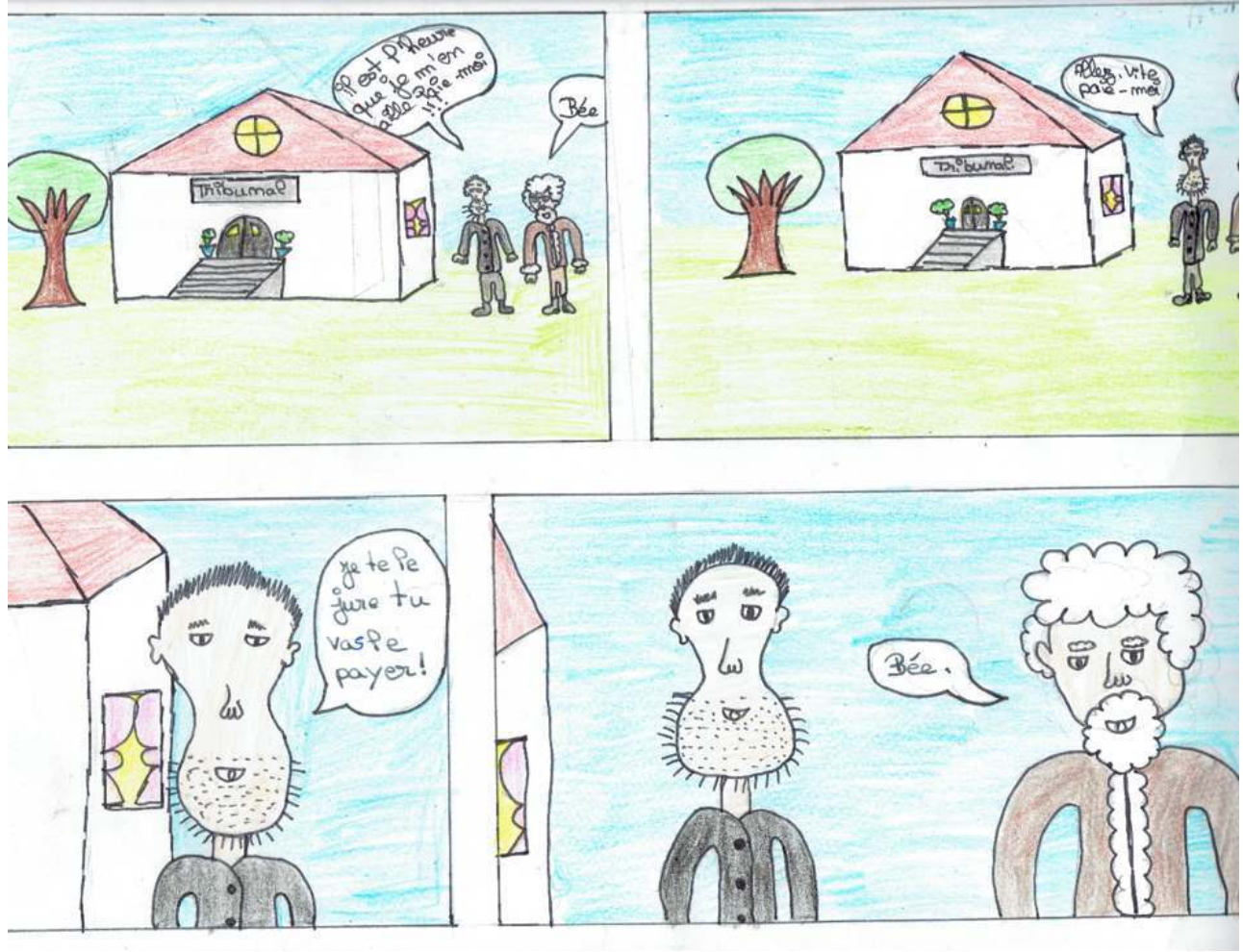

\section{NOTES}

1. Bulletin officiel spécial $n^{\circ} 6$ du 28 août 2008 .

2. Bulletin officiel spécial $n^{\circ} 11$ du 26 novembre 2015 - Les programmes du collège à la rentrée 2016.

3. «Le marché de la bande dessinées et du manga ne bulle pas », 10/02/2016. En ligne : http:// www.presseedition.fr/

le_marche_de_la_bande_dessinee_et_du_manga_ne_bulle_pas_P_AA_R_0_A_13104_html.

4. Deuxième place du top des meilleures ventes avec 274000 exemplaires vendus.

5. «Etude. Les 7-11 ans lisent davantage que les adolescents et les jeunes », 28/06/2016. EN ligne : http://www.ouest-france.fr/culture/livres/etude-les-7-11-ans-lisent-davantage-que-lesadolescents-et-les-jeunes-4331062.

6. Exemples de romans adaptés : L'île au trésor, le Tour du Monde en 80 jours, Robinson Crusoé, NotreDame de Paris, Voyage au Centre de la Terre, L'Odyssée, Le Capitaine Fracasse, Les Misérables (1 et 2), Michel Strogoff, Le Dernier des Mohicans, Guerre et Paix (1 et 2), Tartarin de Tarascon, Germinal (1 et 2), Quo Vadis, Le Rouge et le Noir (1 et 2), Don Quichotte, Le Conte de Noël, Le Livre de la Jungle, Le Monde perdu, des Contes de 1001 nuits, Oliver Twist...

7. «"Romans de Toujours" pour les éditions Adonis », 06/11/2007. En ligne : http://www.bdbest.com/-romans-de-toujours-pour-les-editions-adonis-news-602.html.

8. Le Horla, adaptation de la nouvelle de Guy de Maupassant est parue en mars 2014 et Quatre sœurs, une adaptation d'un roman de M. Ferdjoukh (janv. 2014).

9. 20 titres étaient proposés pour chaque niveau dans les programmes des 1995-1996, plus aucun n'apparait dans les programmes de 2008 où la BD subit le même sort que la littérature de jeunesse, déconsidérée au profit de la littérature patrimoniale.

10. - $65,2 \%$ en sixième et $-71,5 \%$ en cinquième (ibid.). 
11. Bulletin officiel spécial $n^{\circ} 11$ du 26 novembre 2015. Les programmes du collège à la rentrée 2016.

12. En ligne : http://data.bnf.fr/12008638/farce_de_maitre_pierre_pathelin/.

\section{RÉSUMÉS}

Cet article propose de montrer l'intérêt didactique des adaptations transgénériques au collège. Il décrira en particulier l'adaptation d'une farce médiévale $d u X V^{\mathrm{e}}$ siècle au programme de la classe de cinquième, La Farce de Maître Pathelin, dans le genre de la bande dessinée. Outre que cette expérience favorise le travail en projet et en interdisciplinarité français/arts plastiques et donne du sens aux apprentissages par une production finale issue d'un travail par groupes, elle permet de valider des compétences qui ressortissent à tous les domaines du français et de la maitrise de la langue en général (écriture, lecture, langue et oral). Cette séquence s'appuie sur les genres dramatique et verbo-iconique dont il s'agit de s'approprier les codes sémiotiques. Souvent peu valorisés par les programmes scolaires, ils possèdent un fort potentiel d'attractivité pour les élèves de collège, et permettent de réveiller leur motivation. Enfin la transposition dans le mode de la bande dessinée inscrit la pratique dans le parcours artistique et culturel des élèves, en réception comme en production, en leur permettant de découvrir des auteurs, de comparer les techniques et les modes de narration des deux genres ainsi mis en regard.

This article suggests to show the didactic interest of transgeneric adaptation to the secondary school. It will describe in particular, the adaptation of a medieval farce of the 15th century to the program of the second year of middle school, La Farce de Maitre Pathelin, in the genre of the comic strip. Besides that this experience encourages the work in project and in interdisciplinarity French/drawing art, and gives meaning to the learnings by a final production stemming from a work by groups, it allows to validate skills which stood out in all the domains of French and language ability generally (writing, reading, language, oral). This sequence leans on the dramatic and verbo-iconic kinds which it is to appropriate the semiotic codes. Often little valued by the middle school programs, they possess a high potential of attractiveness for the pupils of college, and allow to wake their motivation. Finally, the transposition in the mode of the comic strip registers the practice in the artistic and cultural route of the pupils, in reception as in production, by allowing them to discover authors, to compare techniques and ways of narration of both genres so put in the opposite page.

\section{INDEX}

Mots-clés : théâtre, bande dessinée, adaptation, genre, didactique, pédagogie de projet, compétences en lecture/écriture

Keywords : theater, comic strip, adaptation, genre, didactic, pedagogy of project, skills in read and write 
AUTEUR

ARMELLE HESSE-WEBER

Université de Lorraine, CREM, EA 3476, F-57000, France 